\title{
P-1068- Using Adult Learning Theories in Educational Programs for Healthcare Providers in Kuwait Improved Diabetes Knowledge
}

\author{
Zahraa Rahme ${ }^{1}$, Naglaa Mesbah ${ }^{1}$, Nehad Taha ${ }^{1}$ and Faten Sukkar ${ }^{1}$ \\ ${ }^{1}$ Dasman Diabetes Institute, Education and Training Department, Kuwait
}

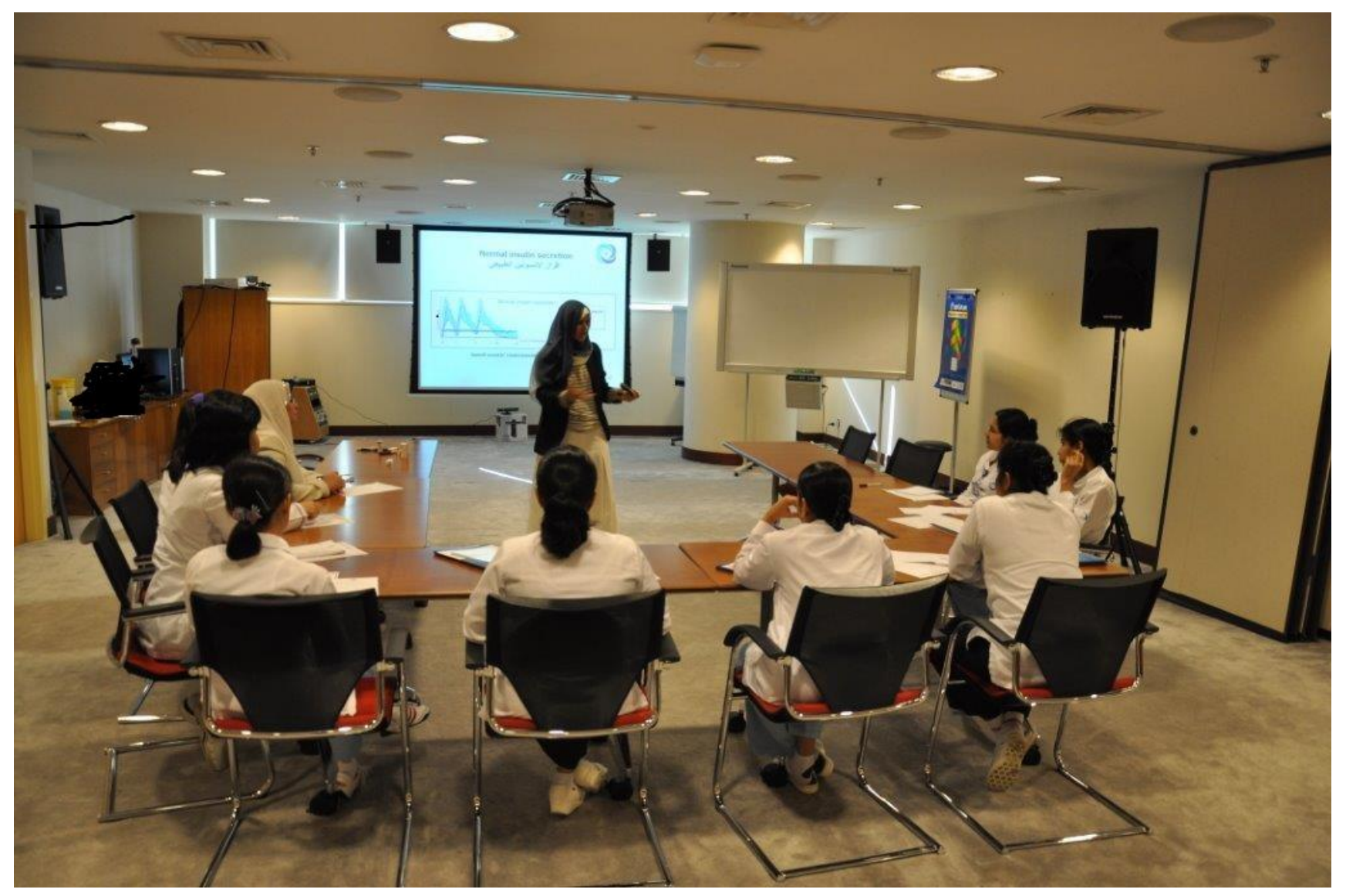

\section{AIM:}

To demonstrate the importance of training the healthcare providers, from multidisciplinary team, with the purpose of guiding them to a better understanding of diabetes and improve their quality of work using adult learning theories.

\section{Methods:}

Basic Diabetes Knowledge and Skills Workshop was planned and delivered by Diabetes Nurse Educators at Dasman Diabetes Institute (DDI). The course was adapted from the International Diabetes Federation (IDF) curriculum for healthcare professionals. The course was planned and implemented using adult theory principles, which can provide useful guidance for medical educators. Forty- two healthcare providers who are novice to diabetes attended this course. The workshop took place over five days, 6 hours per day. Three Certified Diabetes Nurse Educators delivered the course. They used different teaching methods for delivering the contents such as interactive lectures, practical sessions and small group activities. Formative assessments with constructive feedback and evaluation were designed to meet the needs of this workshop. Diabetes Knowledge was assessed using pre and post questionnaires.

\section{Results:}

Score results from pre and post questionnaires were analyzed.

Results demonstrated significant improvement in the participants' knowledge ( $\mathrm{p}$ is $<0.00001$ ). There was positive feedback from participants who expressed the valuable benefit of the workshop. Most of the learners stated that, the sessions met the objectives remarkably well. Also, they expressed satisfaction with the course content and objectives.

\section{Conclusion:}

Significant diabetes knowledge gain resulting from the delivery of this workshop confirmed that implementing adult theories into structured diabetes education for healthcare professionals who are novice to diabetes regardless of their previous experience is an essential component of personal development in order to be able to help people with diabetes in their diabetes selfmanagement

\section{References:}

1. Grant J. Principles of Curriculum Design.In: Swanwick T, Editor. Understanding medical education : evidence, theory and practice. Chichester: Wiley-Blackwell, 2010.

2. Knowles MS. Application in continuing education for the health professions: chapter five of "Andragogy in Action". Mobius 1985;5(2):80-100.

3. Nash M. Mental health nurses' diabetes care skills - a training needs analysis. Br J Nurs 2009;18(10):626, 28-30.

4. el-Deirawi KM, Zuraikat N. Registered nurses' actual and perceived knowledge of diabetes mellitus. J Nurses Staff Dev 2001;17(1):5-11.

5. Bloom BS. Taxonomy of Educational Objectives, etc.

[Edited by B. S. Bloom and others.]: vol. 1. Longmans.

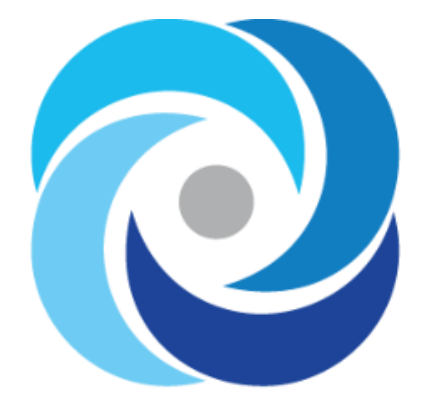

